\title{
DAPT plus anticoagulant therapy: The difficult coexistence post-ACS in older patients with atrial fibrillation
}

\author{
Mauro Di Bari1,2, Alessandra Pratesi1, Francesca M. Nigro1, Irene Marozzi1, \\ Stefano Fumagalli1,2 \\ 1 Research Unit of Medicine of Aging, Department of Experimental and Clinical Medicine, \\ University of Florence \\ 2 Unit of Geriatrics, AOU Careggi, Florence, Italy
}

\begin{abstract}
Atrial fibrillation (AF) and coronary artery disease requiring percutaneous coronary intervention (PCI) and stenting often coexist in older patients. This poses the difficult problem of concurrent anticoagulant and double antiplatelet therapy (triple therapy). Current treatment guidelines do recommend triple therapy, especially in the course of acute coronary syndrome (ACS), with limitations due to an excessive risk of bleeding associated with this therapeutic regimen. This review summarizes randomized clinical trials and observational studies that compared triple therapy with a variety of different therapeutic options. Although the available evidence is not completely satisfactory and other studies are urgently needed, alternative regimens to triple therapy in AF patients undergoing PCI and stenting are promising, at least in terms of safety.
\end{abstract}

\section{Introduction}

Atrial fibrillation (AF) is the most common tachyarrhythmia of late life, affecting approximately $5 \%$ of older persons. Prevalence increases from $4.3 \%$ in subjects aged $66-69$ years to $5.4 \%$ above the age of 90 ; similar age-related trends have been reported for incidence [1]. Episodes

Corresponding author: Prof. Mauro Di Bari, Research Unit of Medicine of Aging, Department of Experimental and Clinical Medicine, University of Florence, Viale Pieraccini 18, 50139 Firenze, Italy.

E-mail: mauro.dibari@unifi.it

Key words: Dual anti-platelet therapy; triple therapy; acute coronary syndrome; atrial fibrillation; older patients, hemorrhagic $v s$ thromboembolic risk.

Received for publication: 26 April 2018

Accepted for publication: 30 May 2018

() Copyright M. Di Bari et al., 2018

Tipografia PI-ME Editrice, Italy

Monaldi Archives for Chest Disease 2018; 88:957

doi: 10.4081/monaldi.2018.957

This article is distributed under the terms of the Creative Commons Attribution Noncommercial License (by-nc 4.0) which permits any noncommercial use, distribution, and reproduction in any medium, provided the original author(s) and source are credited. of clinically unrecognized atrial fibrillation are frequent in individuals with no documented history of the arrhythmia and may be responsible for thromboembolism of unknown origin, which is mostly represented by stroke. Following current guidelines, an oral anticoagulant (OAC) is recommended for patients with $\mathrm{AF}$ and a $\mathrm{CHA}_{2} \mathrm{DS}_{2}$-VASc score $\geq 1$ in men and 2 in women to prevent thromboembolism [2-4].

Coronary artery disease coexists in as many as 30 to $60 \%$ of AF patients and it is estimated that about $5-15 \%$ of AF patients will undergo percutaneous coronary intervention (PCI) and stenting at some point in their lives [2]. As a consequence, the need for triple therapy, i.e. coexistent prescription of $\mathrm{OAC}$, traditionally vitamin $\mathrm{K}$ antagonists (VKA), and dual antiplatelet therapy (DAPT) with aspirin and the $\mathrm{P}_{2} \mathrm{Y}_{12}$ antagonist clopidogrel, often emerges. Although effective in reducing thromboembolic risk, the combination of DAPT with OAC bears a substantial risk of bleeding, which can be suggested by a HAS-BLED score of $\geq 3$ [4]. The balance between stroke and bleeding risks is particularly delicate in older patients, making the issue of triple therapy highly controversial. Moreover, newer therapeutic agents, such as $\mathrm{P}_{2} \mathrm{Y}_{12}$ antagonists more potent than clopidogrel, and non-VKA, direct oral anticoagulants (DOAC) are nowadays available for the prevention of stent thrombosis and AF-related stroke, respectively. Thus, several possible therapeutic scenarios should be considered.

\section{Clinical practice guidelines}

Compared to other current practice guidelines on AF, those issued in 2016 by the European Society of Cardiology (ESC) [2] discuss the topic of triple therapy more extensively. In patients with AF, conventional triple therapy (aspirin 75-100 mg/day, clopidogrel $75 \mathrm{mg} /$ day, and VKA) is recommended for 1 month after elective PCI and for 1 (in case of bleeding high risk) to 6 months (when the risk of bleeding is low) after primary PCI in the setting of ACS, followed by dual therapy (OAC and only one antiplatelet agent) for the time being through 1 year, then by OAC monotherapy. Treatment with any OAC plus clopidogrel $75 \mathrm{mg} /$ day is deemed as an alternative to initial therapy in selected patients. Yet, these guidelines were issued without an undisputable evidence support: randomized clinical trials (RCT) are scarce and, if ever, cast doubts on triple therapy as the most appropriate treatment in $\mathrm{AF}$ patients receiving PCI after ACS.

\section{Evidence from randomized clinical trials}

Only three RCT have been conducted, two before and one after the publication of the ESC guidelines. The WOEST study enrolled 573 patients on warfarin and PCI to warfarin plus DAPT versus a double 
therapy regimen of warfarin plus clopidogrel. After a 1-year follow-up, the primary endpoint of any bleeding episode was reached in $44.4 \%$ of participants in the triple therapy group, compared to only $19.4 \%$ in the double therapy group, with an HR (95\% CI) of 0.36 (0.26-0.50). Somehow unexpectedly, also the secondary endpoints of all-cause death (triple: $6.3 \%$, double: $2.5 \%$ ) and a composite endpoint of death, myocardial infarction, stroke, target-vessel revascularization, and stent thrombosis (triple: $17.6 \%$, double: $11.1 \%$ ) were significantly prevented by the double therapy, with HR of $0.39(0.16-0.93)$ and 0.60 (0.38-0.94) [5]. Thus, the combination of warfarin and clopidogrel appeared more effective and safer than triple therapy. Nevertheless, applicability of these findings to AF patients treated with PCI for ACS should be cautious, because only $70 \%$ of participants in the WOEST trial were on warfarin for AF and a minority (30\%) had received PCI in the course of ACS.

The PIONEER RCT opened a new scenario, by introducing a DAOC as a comparator to VKA in a combined anticoagulant-antiplatelet therapeutic schedule. A total of 2024 patients, all with AF, were randomized to three therapeutic regimens, i.e. rivaroxaban $15 \mathrm{mg} /$ day plus a $\mathrm{P}_{2} \mathrm{Y}_{12}$ antagonist (group 1), rivaroxaban at the unusually low dose of $5 \mathrm{mg}$ /day plus DAPT (group 2), and VKA plus DAPT (group 3). Clopidogrel was the $\mathrm{P}_{2} \mathrm{Y}_{12}$ antagonist used in approximately $95 \%$ of cases. After 1-year follow-up, bleeding episodes were significantly less frequent in groups 1 and 2 than in group 3, with HR of 0.59 (0.47-0.76) and $0.63(0.50-0.80)$, respectively, and incidence of bleeding of $17.4 \%$ in the two groups receiving rivaroxaban combined, versus $26.7 \%$ in group 3 . Incidence of major cardiovascular events was comparable across the groups. Groups 1 and 2 had similar incidence of both safety and efficacy endpoints. These findings indicate that rivaroxaban, associated with a $\mathrm{P}_{2} \mathrm{Y}_{12}$ antagonist or with DAPT, is better than warfarin in terms of bleeding risk and not inferior for efficacy [6]. Strength of the study was enrolment of AF patients only, whereas a limitation was that the standard dose of rivaroxaban used for AF is 20 and not $15 \mathrm{mg} / \mathrm{day}$, which was selected in order to reduce risk of bleeding.

In the more recent RE-DUAL PCI trial, Cannon et al. [7] evaluated a different DAOC, dabigatran, randomly assigning 2725 patients with $\mathrm{AF}$ and PCI to three possible therapeutic alternatives: dual therapy with dabigatran (110 mg twice daily) plus either clopidogrel (75 mg daily) or ticagrelor ( $90 \mathrm{mg} /$ day), dual therapy with dabigatran (150 mg twice daily) plus either clopidogrel or ticagrelor, or triple therapy with warfarin plus aspirin ( $\leq 100 \mathrm{mg}$ daily) and either clopidogrel or ticagrelor. In the triple therapy group, aspirin was discontinued after 1 or 3 months, depending on whether a bare-metal or a drug-eluting stent had been implanted. Primary endpoint was bleeding, whereas a main secondary efficacy endpoint combined thromboembolic events (myocardial infarction, stroke, or systemic embolism), death, or unplanned revascularization (either interventional or surgical). Compared to triple therapy, incidence of the primary safety endpoint was lower in both dual therapy arms, with HR of $0.52(0.42-0.63)$ and 0.72 (0.58-0.88), respectively; combined, the two dabigatran-based dual therapy regimens conferred the same protection as triple therapy towards the secondary efficacy endpoint, with HR of 1.04 (0.84-1.29) [7]. However, it should be taken into account that in the RE-DUAL PCI study indication to PCI was ACS in approximately $50 \%$ of cases only.

Taken together, the results of these three randomized trials suggest that newer, diverse therapeutic approaches, which reject the paradigm of aspirin and VKA as the cornerstones to prevent thromboembolism in PCI with stenting and AF, respectively, are at least as effective and definitively safer than the time-honored triple therapy in patients with $\mathrm{AF}$ undergoing PCI, although not always in the context of ACS.

\section{Other evidence}

Observational studies, in general deriving from clinical registries, are frequently considered to complement, from the "real-world", evidence provided by RCT. In a registry study of 12165 patients with AF, undergoing primary PCI of ACS, Lamberts et al. reported that triple therapy was associated with a non-significant increase in both risk of bleeding (HR 1.28, 95\% CI 0.89-1.82) and of myocardial infarction or coronary death (HR 1.45, 95\% CI 1.00-2.08) in 1 year [8]. The risk of bleeding, but not that of thromboembolism, was significantly greater with triple therapy in a registry study of approximately 5000 patients [9] and in a meta-analysis of more than 7100 patients [10], in which this treatment was compared with clopidogrel-based dual therapy.

Data on newer $\mathrm{P}_{2} \mathrm{Y}_{12}$ antagonists are usually more limited in terms of sample size, follow-up duration, and design [11, 12]: however, in none of the available investigations, including a large $(\mathrm{n}=11756)$ observational study comparing prasugrel plus OAC vs. clopidogrel plus OAC in a 6-month follow-up [12], triple therapy was more effective or safer than the comparator.

Two other meta-analyses addressing this theme have been conducted. In the first one, 14 observational studies (including conference proceedings) and 2 RCT were pooled, comparing triple therapy with different regimens of OAC (given with a variety of indications besides AF) associated with one single antiplatelet agent [13]. Mortality and other efficacy endpoints were less frequent in subjects receiving triple therapy, although with a substantial degree of heterogeneity that makes the result of the study uncertain; bleeding was significantly more common in subjects receiving triple therapy. Safety of this regimen was inferior, with neutral findings on the efficacy endpoints, in another meta-analysis that included 9 observational studies and 2 RCT [14].

\section{Conclusions}

Evidence does not unequivocally support the use of the guideline-recommended triple therapy in patients with AF and recent PCI for ACS. From the 3 RCT available, alternative regimens based on clopidogrel plus VKA, or a DOAC plus different antiplatelet agents, are at least as effective and definitively safer in patients with diverse indications to PCI and stenting. If this evidence can be strictly applied in the setting of ACS, although reasonable, remains so far unproven. Observational studies seem to confirm, in a real-world setting, that alternatives to triple therapy are safer and possibly non-inferior. Meta-analyses had poor methodological quality and reported conflicting results on efficacy, whereas they confirmed that safety of triple therapy was lower than that of the comparators. The available evidence, although not adequate to indicate a definitively preferable alternative, appear sufficient to question the totem of triple therapy in AF patients undergoing PCI and stenting, at least for safety reasons. For the practicing physician, it is also important to recognize that avoiding triple therapy may help reduce the complexity of a therapeutic regimen, thus contributing to limit problems such poor drug adherence and drug overload (polypharmacy), typical of older patients.

As summarized by Chang et al. [15], at least 6 RCT are currently ongoing, comparing a variety of therapeutic options (mostly based upon DAOC) to triple therapy. Of them, the APPROACH study will specifically focus on patients with ACS, who are randomized to apixaban plus clopidogrel versus triple therapy. While the results of this and other studies are urgently needed, it should be pointed out that none of the available investigations and, as far as it can be argued from the design studies, also of those under way, considered outcomes other than death, cardiovascular events, and clinically significant bleedings. Even "minor" 
bleedings, as well as subclinical cerebrovascular events, may induce a substantial decline in functional and cognitive status of older persons, who are frequent candidates to triple therapy. Moreover, age-related frailty [16] may disproportionately amplify the negative effects of thromboembolism and bleeding. Thus, evidence so far provided on the issue of efficacy and safety of triple therapy and alternatives to it is largely unsatisfactory in terms of specific geriatric outcomes.

\section{References}

1. Piccini JP, Hammill BG, Sinner MF, et al. Incidence and prevalence of atrial fibrillation and associated mortality among Medicare beneficiaries, 1993-2007. Circ Cardiovasc Qual Outcomes 2012;5:85-93.

2. Kirchhof P, Benussi S, Kotecha D, et al. 2016 ESC guidelines for the management of atrial fibrillation developed in collaboration with EACTS: the Task Force for the management of atrial fibrillation of the European Society of Cardiology (ESC) developed with the special contribution of the European Heart Rhythm Association (EHRA) of the ESC Endorsed by the European Stroke Organisation (ESO). Eur Heart J 2016;37:2893-962.

3. Kirchhof P, Ammentorp B, Darius H, et al. Management of atrial fibrillation in seven European countries after the publication of the 2010 ESC guidelines on atrial fibrillation: primary results of the PRE- vention of thromboemolic events-European Registry in Atrial Fibrillation (PREFER in AF). Europace 2014;6:6-14.

4. Lamberts M, Olesen JB, Ruwald MH, et al. Bleeding after initiation of multiple antithrombotic drugs, including triple therapy, in atrial fibrillation patients following myocardial infarction and coronary intervention: a nationwide cohort study. Circulation 2012;126:1185-93.

5. Dewilde WJ, Oirbans T, Verheugt FW, et al. Use of clopidogrel with or without aspirin in patients taking oral anticoagulant therapy and undergoing percutaneous coronary intervention: an openlabel, randomised, controlled trial. Lancet 2013;381:1107-15.

6. Gibson CM, Mehran R, Bode C, et al. An open-label, randomized, controlled, multicenter study exploring two treatment strategies of rivaroxaban and a dose-adjusted oral vitamin $\mathrm{K}$ antagonist treatment strategy in subjects with atrial fibrillation who undergo percutaneous coronary intervention (PIONEER AF-PCI). Am Heart J 2015;169:472-8.

7. Cannon CP, Bhatt DL, Oldgren J, et al. Dual Antithrombotic therapy with dabigatran after PCI in atrial fibrillation. N Engl J Med 2017;377:1513-24.

8. Lamberts M, Gislason GH, Olesen JB, et al. Oral anticoagulation and antiplatelets in atrial fibrillation patients after myocardial infarction and coronary intervention. J Am Coll Cardiol 2013;62:981-9.

9. Hess CN, Peterson ED, Peng SA, et al. Use and outcomes of triple therapy among older patients with acute myocardial infarction and atrial fibrillation. J Am Coll Cardiol 2015;66:616-27.

10. D’Ascenzo F, Taha S, Moretti C, et al. Meta-analysis of randomized controlled trials and adjusted observational results of use of clopidogrel, aspirin, and oral anticoagulants in patients undergoing percutaneous coronary intervention. Am J Cardiol 2015;115:1185-93.

11. Braun 0Ö, Bico B, Chaudhry U, et al. Concomitant use of warfarin and ticagrelor as an alternative to triple antithrombotic therapy after an acute coronary syndrome. Thromb Res 2015;135:26-30.

12. Jackson LR 2nd, Ju C, Zettler M, et al. Outcomes of patients with acute myocardial infarction undergoing percutaneous coronary intervention receiving an oral anticoagulant and dual antiplatelet therapy: A comparison of clopidogrel versus prasugrel from the TRANSLATE-ACS study. JACC Cardiovasc Interv 2015;8:1880-9.

13. Barbieri L, Verdoia M, Schaffer A, et al. Risk and benefits of triple therapy in patients undergoing coronary stent implantation requiring oral anticoagulation: A meta-analysis of 16 studies. Cardiovasc Drugs Ther 2016;30:611-22.

14. Agarwal N, Jain A, Mahmoud AN, et al. Safety and efficacy of dual versus triple antithrombotic therapy in patients undergoing percutaneous coronary intervention. Am J Med 2017;130:1280-9.

15. Chang KW, Arbit B, Hsu JC. Antithrombotic regimens in patients with atrial fibrillation and coronary artery disease after percutaneous coronary intervention: A focused review. Int J Cardiol 2017;243:263-9.

16. Di Bari M, Balzi D. Cardiovascular disease in the oldest old: a geriatric epidemiology perspective. In: Ungar A and Marchionni N, Editors. Cardiac management in the frail elderly patient and the oldest old. Elsevier; 2017; p. 9-22. 